\title{
Adding the PubMed unique identifier to journal article references without increasing the bulkiness of the journal
}

\author{
Subhash Chandra ${ }^{1}$, Melissa L. Rethlefsen ${ }^{2}$, Brian W. Pickering ${ }^{3}$, J. Michael Homan², \\ Ognjen Gajic ${ }^{4}$, Vitaly Herasevich ${ }^{3}$
}

${ }^{1}$ Department of Emergency Medicine, Mayo Clinic Rochester, MN

${ }^{2}$ Mayo Clinic Libraries, Mayo Clinic, Rochester, MN

${ }^{3}$ Department of Anesthesiology, Mayo Clinic Rochester, MN

${ }^{4}$ Department of Internal Medicine, Division of Pulmonary and Critical Care Medicine, Mayo Clinic Rochester, MN

Corresponding author: Vitaly Herasevich Department of Anesthesiology

College of Medicine, Mayo Clinic

2001 1st St SW, Rochester, MN 55905, USA

herasevich.vitaly@mayo.edu

Tel.: + 15072554055

Fax: + 15072554267

Received: 9 April 2011

Accepted: 4 November 2011

Copyright (C) 2011 by Academy of Sciences and Arts of Bosnia and Herzegovina. E-mail for permission to publish:amabih@anubih.ba

Nowadays a large amount of scientific and medical literature can be accessed online in full text format. Although the manuscripts are widely available online, the readers' preference is often for a printed version through personal or library print subscriptions or by printing a copy of the online version for reading convenience.

We propose the idea that adding the PubMed Unique Identifier (PMID) to every PubMedindexed citation in biomedical journal articles' reference lists will simplify article retrieval by providing a short and unique search string to use with internet search engines. By determining the empty spaces at the end of a set of journal articles and calculating the space required to accommodate the addition of PMIDs for each reference, we tested the hypothesis that adding a PMID to references will not increase the printed size of a journal.

We selected 18 clinical journals in the Mayo Clinic Library, covering most major publishers. One issue of each selected journal was randomly picked from library bookshelves within a range of one year (November-2008 to November-2009). The primary outcome was the increase in number of printed pages for references after adding PMIDs. The space occupied or left blank was measured using a transparent template divided into 20 equal portions, each portion representing $5 \%$ of the page.

We analyzed 280 articles from 18 journal issues. The median numbers of articles per issue was 13 , ranging from 5 to 38 . The number of references per article varied widely from 7 to 199 , and the median was 30 references per article. Overall, $92 \%$ of references were included in PubMed. The amount of empty space at the end of every article differed between individual journals with an average of $45 \%$ (95\% CI; $40 \%$ to $48 \%)$ of empty space.

If the PMID is added to all PubMed references, (generally an eight digit number for current citations) it would increase the length of each article by a median (interquartile range) of $3 \%$ ( $2 \%$ to $4 \%)$. Journals would still have an average of $40 \%$ (95\% CI; $35 \%$ to $45 \%$ ) of empty space following each reference list.

Our results suggest that adding a PMID to each cited reference will not substantially increase the length of a printed journal. While the effect of adding the PMID to reference lists in printed publications is minimal, the feature will save readers time in retrieving reference articles and make this process more convenient to users.

Key words: PubMed Unique Identifier, References, Usability. 